\title{
A solution of two-person single-suit whist
}

\author{
Johan Wästlund \\ Department of Mathematics \\ Linköping University, Linköping, Sweden \\ jowas@mai.liu.se \\ Submitted: May 21, 2001; Accepted: Aug 29, 2005; Published: Sep 5, 2005 \\ Mathematics Subject Classification: 91A46
}

\begin{abstract}
We give a complete solution of the combinatorial game of two-person single-suit whist. This game is played with a deck consisting of a single totally ordered suit of $2 n$ cards. Each of the two players receives $n$ cards. Hence both players have complete information about the distribution of the cards. One of the players is said to be on lead. Play proceeds in rounds called tricks. The player on lead plays one of his cards, and with knowledge of this card, the other player plays one of his cards. The player with the higher card wins the trick, and obtains the lead. The cards that are played are then removed. Play continues until all cards are exhausted. Each player tries to win as many tricks as possible.

Our solution provides an efficient algorithm for calculating the game theoretical value of any distribution of the cards.
\end{abstract}

\section{Introduction}

\section{$1.1 \quad$ Trick taking games}

Playing cards were probably introduced in Europe around 1370. According to D. Parlett [8], simple trick taking games for two players were around at least as early as in the beginning of the fifteenth century. It is therefore reasonable to assume that this family of games are among the oldest games played with a deck of cards.

Card games, and in particular trick taking games, have remained among the most popular and commonly played social games ever since. Today, the family of trick taking games includes a large variety of games for different numbers of players, among them the game of bridge, by many considered the most intellectually challenging of all card games.

Trick taking games have certain fundamental rules in common. Each player is dealt the same number of cards, and the cards are played in rounds called tricks, consisting of one card from each player. In each trick, the player who plays first is said to have the lead, and his card has a special status. The other players have to play a card in the same 
suit, to follow suit, if they have such a card. The player who plays the highest card in this suit wins the trick (unless the game is played with trumps, but we will not consider trumps here), and plays first in the following trick.

The rules for scoring differ from game to game. In the traditional forms of the game, which we refer to as whist, the goal is simply to take as many tricks as possible. In modern forms such as bridge, the first phase of the game consists of an auction, where the side who makes the final bid gets the contract. The main objective in the second phase of the game, the card play, is for the side who made the final bid to make their contract, that is, to take at least a certain number of tricks determined by the final bid.

In the so called misère games, the object is to take as few tricks as possible, or as in Hearts, to avoid taking tricks that contain certain cards.

Femkort (Five-card) is a game which is common in Sweden, but does not seem to be well known internationally. As the name suggests, each player starts with five cards. In this game, the winner is the player who takes the last trick. Together with its misère counterpart Gurka (Cucumber), it shares what we here refer to as the greedy rule. Not only does a player have to follow suit, but he is also forced to play a higher card than the highest previously played card in this suit, if he can.

\subsection{Two-person single-suit trick taking games}

It is natural from a mathematical point of view to consider whist played with a single suit. Most bridge players, when planning declarer play, seem to regard the game roughly as a "sum" of its four single-suit subgames, in the spirit (although not strictly according to the definition) of combinatorial game theory. This can be seen from the way the declarer makes a preliminary estimate of the number of tricks he will be able to take by summing the number of tricks he can take in each suit. In this paper, we only consider games played with a single suit. Some results on two-person whist with more than one suit can be found in [9].

As a guide to advanced techniques of card play, the book [7] by G. Ottlik and H. Kelsey has become a classic. In fact it was the original and very scientific style of this book that inspired me to take up the research that led to this paper. For an introduction to combinatorial game theory, we refer to [1].

We consider trick taking games played with a single suit consisting of $2 n$ cards. We may number the cards from 1 to $2 n$, where 1 is the smallest card, and $2 n$ is the highest. When $n$ is sufficiently small $(n \leq 6)$ it is convenient to use the traditional numbering from 2 to 10, Jack, Queen, King and Ace.

We study three different trick taking games. All three have the following rules in common: The cards are distributed (perhaps randomly) between the two players East and West, so that each player receives $n$ cards. Hence both players have complete information about the distribution of the cards. The game consists of $n$ tricks. In each trick, the players take turns playing one of their cards. The player who played the highest card wins the trick, and plays first in the next trick.

Of the three games we consider, Whist is the one with the simplest rules, and at the 
same time, the one whose analysis turns out to be most difficult. The object of Whist is to win as many tricks as possible.

The problem of determining the game theoretical value of single-suit whist was posed in 1929 by the mathematician and chess world champion Emanuel Lasker [5, 6]. The study of the game was continued by J. Kahn, J. C. Lagarias and H. S. Witsenhausen $[2,3]$, who also solved the misère form of the game [4].

In Greedy Whist, the object is still to take as many tricks as possible, but this game is played with the greedy rule, restricting the choice for the player who does not have the lead. Hence in each trick, the player who plays second must take the trick if he can. It turns out that this additional rule simplifies the analysis considerably.

The game of Five-card is also played with the greedy rule. However, the winner is the player who wins the last trick. We will refer to this game as Five-card although we consider the generalization to $n$ cards on each hand.

The game here called Whist is of course just a special case of the ordinary game of whist, modeling an endgame with only two players and one suit involved. In the same way, Five-card is the two-person single-suit counterpart of the real-world game. Greedy Whist is a somewhat artificial cross-breed of the two. I do not know whether such a game (or rather its counterpart with an ordinary deck of cards) is actually played, although the greedy rule occurs in several card games. This game is interesting from a theoretical point of view, since it is simple enough to allow a fairly straightforward solution, yet provides a good approximation to Whist.

\section{Examples}

We illustrate the three games with a few simple examples.

\subsection{Five-card}

$\begin{array}{ll}\text { West : } & \text { East : } \\ \mathrm{KQ} & \text { A J }\end{array}$

If East has the lead, he will play the jack, saving the ace for the last trick. On the other hand, if West has the lead, East will have to win the first trick with the ace, and West will take the last trick. Hence in this position, the player on lead has a winning strategy.

\subsection{Greedy Whist}

In single-suit whist, it is in general a disadvantage to have the lead. The following is a standard ending:

$$
\begin{array}{ll}
\text { West : } & \text { East }: \\
\mathrm{K} \mathrm{J} & \mathrm{A} \mathrm{Q}
\end{array}
$$

Here, the ace will of course always win a trick. Whether West gets a trick or not depends on the location of the lead. If West has the lead, East will take both tricks. If 
East has the lead however, West can always make sure to play his king in the same trick as East's queen.

The following two examples show that it is sometimes correct to lead a high card, sometimes correct to lead a small one:

$$
\begin{array}{ll}
\text { West : } & \text { East : } \\
\text { K 10 } 9 & \text { A Q J }
\end{array}
$$

Here West on lead will get one trick, provided he leads the nine or ten.

$$
\begin{array}{ll}
\text { West : } & \text { East : } \\
\text { Q J } 9 & \text { A K } 10
\end{array}
$$

Again West on lead should get one trick. Here on the other hand, it is essential to lead a high card, the queen or the jack, in order not to give East a cheap trick with the ten. The three examples above are equally valid for relaxed (non-greedy) whist.

\section{$2.3 \quad$ Whist}

We point out that the right not to take a trick can be valuable. This means that there is an essential difference between greedy and relaxed whist.

$$
\begin{array}{ll}
\text { West : } & \text { East }: \\
\text { K Q } 10 & \text { A J } 9
\end{array}
$$

If West leads the king and East wins with the ace, West will get the last two tricks. On the other hand, if East is allowed to let West keep the first trick, East recovers this trick with interest, as he then keeps A J against West's Q 10, with West on lead. Hence with West on lead, East will take two tricks in Relaxed Whist, but only one trick in Greedy Whist. The method of playing low to gain a trick later is called holding up. The example (5) is known to experienced bridge players. It is the only example of its kind that arises with some frequency at the bridge table (although in bridge it is quite common to hold up for entirely different reasons).

\section{Mathematical representation}

A card distribution or deal is a partition of a totally ordered set of $2 n$ elements, the cards, into two $n$ element subsets called the East and West hands. Let $D$ be a deal. We denote the West cards by

$$
W_{1}(D)<W_{2}(D)<\cdots<W_{n}(D)
$$

and similarly, we let East's cards be

$$
E_{1}(D)<E_{2}(D)<\cdots<E_{n}(D) .
$$

When discussing a particular deal, we suppress the dependence on $D$ and write $W_{i}=$ $W_{i}(D)$ and $E_{j}=E_{j}(D)$. 
where

We represent the distribution of the cards by an $n$ by $n$ matrix $A=A(D)=\left(A_{i j}\right)$,

$$
A_{i j}= \begin{cases}1, & \text { if } W_{i}>E_{j} \\ -1, & \text { if } W_{i}<E_{j} .\end{cases}
$$

We show in Section 7.1 that the trace of this matrix determines the outcome of Fivecard under optimal play. In the analysis of Whist and Greedy Whist, we consider a more general sum along diagonals of $A$. For $-n<k<n$, we let

$$
T_{k}=T_{k}(D)=\sum_{\substack{1 \leq i, j \leq n \\ j-i=k}} A_{i j} .
$$

Hence $T_{0}=\operatorname{tr}(A)$.

For example, the deal (5) is represented by the matrix

$$
A=\left(\begin{array}{ccc}
1 & -1 & -1 \\
1 & 1 & -1 \\
1 & 1 & -1
\end{array}\right)
$$

and $T_{-2}=1, T_{-1}=2, T_{0}=1, T_{1}=-2$ and $T_{2}=-1$.

Notational convention. Several proofs in this paper proceed by induction on $n$, the number of cards on each hand. Hence we need to consider the situation after the first trick, and to verify that certain hypotheses are satisfied. When doing so, we will use primed symbols. For example, $A^{\prime}=A\left(D^{\prime}\right)$ denotes the matrix corresponding to the distribution of the cards that remain after the first trick. Notice that the primed symbols depend not only on the original distribution $D$ of the cards, but also on the play in the first trick.

\section{The normal strategy}

In Sections 6, 7.1 and 8, we study the three games Five-card, Greedy Whist, and Whist. We give complete solutions to the first two games, and for the third, a value which differs by at most one trick from the game theoretical value (a complete solution of Whist is given in Section 9). Remarkably, these three results are obtained by one and the same strategy. In this section, we describe this strategy, which will be called the normal strategy. This strategy is hence optimal for both Five-card and Greedy Whist, while in Whist, it scores at most one trick worse than optimal play.

\subsection{Normal strategy for playing second}

When playing second in a trick: If possible, play the smallest card that will win the trick. Otherwise, play the smallest card.

In Five-card and Greedy Whist, taking the trick is obligatory. In this case the normal strategy just means playing the smallest card possible. It is perhaps not surprising that 
this turns out to be optimal. In Whist, taking the trick is not obligatory, and as we have already seen, not always optimal. However, it has been shown that even in Whist, either it is optimal to take the trick as cheaply as possible, or it is optimal to play the smallest card $[2]$.

\subsection{The normal lead}

The lead is the nontrivial part of the normal strategy. Suppose that West has the lead. If all West's cards are higher than all East's cards, then West will take the remaining tricks regardless of his lead. If not all West's cards are high, we choose $i$ and $j$ such that $A_{i, j}=-1$, with the difference $j-i$ as small as possible. Then we let West lead the card $W_{i}$. Similarly, if East has the lead, then we choose $i$ and $j$ such that $A_{i, j}=1$, with $i-j$ as small as possible. East then leads the card $E_{j}$.

In the following sense, this is a "greedy" choice.

Proposition 4.1. Assume that West has the lead, and that East is known to follow the normal strategy for playing second. Then the normal lead maximizes the sum of the entries in $A^{\prime}$.

Proof. Playing a high card obviously minimizes the sum of the entries in $A^{\prime}$, so we need not consider this. Hence if West plays the card $W_{i}$, then East will take the trick with the card $E_{j}$, where $j$ is chosen minimal under the condition that $E_{j}>W_{i}$. In other words, given the choice of $i$, East will choose $j$ such that $j-i$ is minimized under the condition $A_{i, j}=-1$. $A^{\prime}$ is then obtained by deleting row $i$ and column $j$ from $A$. Row $i$ has negative entries to the right of and including $A_{i, j}$, while column $j$ has negative entries above and including $A_{i, j}$. It makes no difference if we assume that the card $W_{i}$ is largest in its sequence, so that West has no card between $W_{i}$ and $E_{j}$. In this case all entries below and to the left of $A_{i, j}$ are positive. Hence when deleting row $i$ and column $j$, we delete $n-1+j-i$ positive entries, and $n+i-j$ negative ones. We clearly maximize the sum of the entries in $A^{\prime}$ by deleting as few positive entries as possible. We do this by minimizing $n-1+j-i$, that is, by minimizing $j-i$.

\section{Some preliminary results}

If both players follow the normal strategy, then for most of the time, the player playing second will take the trick as cheaply as possible, that is, with the smallest card which is higher than the card that was led. The following lemma determines how this affects $T_{k}$ for various $k$. If several consecutive cards belong to the same player, these cards are equivalent. We can therefore assume that the player on lead always leads the highest card in a sequence. We also note that this is consistent with the normal strategy.

Lemma 5.1. Suppose one player leads a card which is highest in its sequence, and the other player takes the trick as cheaply as possible. Suppose that the cards played in this 
trick are $W_{i}$ and $E_{j}$ (so that with the standard numbering of the cards, either $E_{j}=W_{i}+1$ or $\left.E_{j}=W_{i}-1\right)$. Then, for $-n+1<k<n-1$,

$$
T_{k}^{\prime}= \begin{cases}T_{k}+1, & \text { if } k>j-i \\ T_{k}-1, & \text { if } k<j-i \\ T_{k}-A_{i, j}, & \text { if } k=j-i .\end{cases}
$$

Moreover,

$$
A_{p, q}^{\prime}= \begin{cases}A_{p, q}, & \text { if } p<i, \\ A_{p+1, q+1}, & \text { if } p \geq i .\end{cases}
$$

Proof. By the choice of $i$ and $j, A_{p, q}$ is positive whenever $p \geq i, q \leq j$, and at least one of the inequalities is strict. Similarly, $A_{p, q}$ is negative whenever $p \leq i$ and $q \geq j$, and at least one of the inequalities is strict. This implies equation (7).

Let $S$ be the set of matrix positions that do not occur in the right hand side of (7), that is,

$$
S=\{(n, 1),(n-1,1), \ldots,(i, 1),(i, 2), \ldots,(i, n),(i-1, n), \ldots,(1, n)\} .
$$

Then

$$
T_{k}^{\prime}=T_{k}-\sum_{\substack{(p, q) \in S \\ q-p=k}} A_{p, q}
$$

The sum in (8) consists of only one term, and this term is negative if $k>j-i$, positive if $k<j-i$, and equal to $A_{i, j}$ if $k=j-i$.

Note that by symmetry, we can strengthen the conclusion to

$$
A_{p, q}^{\prime}= \begin{cases}A_{p, q}, & \text { if } p<i \text { or } q<j \\ A_{p+1, q+1}, & \text { if } p \geq i \text { or } q \geq j\end{cases}
$$

The following Lemma implies that, apart from the trivial special cases where one player's cards are all higher than the other player's cards, $T_{k}$ changes sign from positive to negative exactly once.

Lemma 5.2. There are numbers $r$ and $s,-n \leq r<s \leq n$, such that $T_{k}=n+k$ if $k \leq r$, $T_{k}=-n+k$ if $k \geq s$, and $T_{k}$ is strictly decreasing on the interval $r \leq k \leq s$.

Proof. If $j-i>0$ and $A_{i j}=1$, then $A_{i, j-1}=A_{i+1, j}=1$. Now suppose that $k>0$ and $T_{k}>-n+k$, so that $A_{i, j}=1$ for at least one entry along the diagonal $j-i=d$. Then for every such value of $i$ and $j$ contributing +1 to $T_{k}$, there is a corresponding term $A_{i, j-1}=1$ contributing +1 to $T_{k-1}$. Moreover, for the largest value of $i$ for which $A_{i, i+k}=1$, there is also a term $A_{i+1, i+k}=1$ contributing to $T_{k-1}$. Since the sum for $T_{k-1}$ has only one more term than that of $T_{k}$, and strictly more positive terms, we conclude that $T_{k-1}>T_{k}$.

Similarly, if $k<0$ and $T_{k}<n+k$, then $T_{k+1}<T_{k}$. This proves the lemma. 
We let the numbers $\bar{H}$ and $\underline{H}$ define the point where $T_{k}$ changes sign, in the following way:

$$
\begin{cases}\bar{H}=\underline{H}=n, & \text { if all West's cards are high, } \\ \bar{H}=\underline{H}=-n, & \text { if all East's cards are high, } \\ \bar{H}=\underline{H}=k, & \text { if } T_{k}=0, \\ \underline{H}=k \text { and } \bar{H}=k+1, & \text { if } T_{k}>0 \text { and } T_{k+1}<0 .\end{cases}
$$

\section{Solution of Greedy Whist}

In this section, we determine the game theoretical value of Greedy Whist. It turns out that this value can be described in terms of $\bar{H}$ and $\underline{H}$. We let

$$
H_{\text {Greedy }}= \begin{cases}\bar{H}, & \text { if } \quad \bar{H}>0, \\ \underline{H}, & \text { if } \quad \underline{H}<0, \\ 0, & \text { if } \quad \bar{H}=\underline{H}=0 .\end{cases}
$$

$H_{\text {Greedy }}$ determines the game theoretical value of Greedy Whist in the following sense:

Theorem 6.1. In Greedy Whist, the normal strategy is optimal. Moreover, the number of tricks that West gets under optimal play is given by the number

$$
\frac{n+H_{\text {Greedy }}}{2} .
$$

If this number is not an integer, it should be rounded down if West has the lead, and up if East has the lead.

Note that by interchanging the roles of East and West, it follows that East is guaranteed

$$
\frac{n-H_{\text {Greedy }}}{2}
$$

tricks, rounded up if West has the lead, and down if East has the lead. In other words, East should take the remaining tricks. Hence in order to prove Theorem 6.1, we only have to show that West is guaranteed the stated number of tricks using the normal strategy.

Proof of Theorem 6.1. We proceed by induction on $n$. We consider three cases.

Case 1: West is on lead. If all West's cards are high, he will take $n$ tricks, which was to be proved. We therefore assume that West has a card which is smaller than one of East's cards, in other words, that there is a negative entry in $A$. We have to show that West can take at least

$$
\left\lfloor\frac{n+H_{\text {Greedy }}}{2}\right\rfloor
$$


tricks. West will not play a high card. Therefore East will win and be on lead after the first trick. By induction, West will be able to take

$$
\left\lceil\frac{n-1+H_{\text {Greedy }}^{\prime}}{2}\right\rceil=\left\lfloor\frac{n+H_{\text {Greedy }}^{\prime}}{2}\right\rfloor
$$

tricks. Hence it suffices for West to make sure that $H_{\text {Greedy }}^{\prime} \geq H_{\text {Greedy }}$.

Following the normal strategy, we let $i$ and $j$ be such that $A_{i, j}=-1$, with $j-i$ as small as possible, and let West play the card $W_{i}$. We can now assume that East takes the trick with the card $E_{j}$, since this will minimize each entry of $A^{\prime}$, and thereby minimize $T_{k}^{\prime}$ for every $k$.

By Lemma 5.1, $T_{k}^{\prime}=T_{k}+1$ if $k \geq j-i$. On the other hand, if $k<j-i$, then by the choice of $i$ and $j$, all the terms contributing to $T_{k}^{\prime}$ are positive. This shows that for $-n+1<k<n-1$,

$$
T_{k}^{\prime} \geq \min \left(1, T_{k}+1\right)
$$

It follows that $H_{\text {Greedy }}^{\prime} \geq H_{\text {Greedy }}$.

Case 2: East is on lead, and leads a high card. If East leads a high card, West will play his smallest card. This means that $A^{\prime}$ is obtained by deleting the first row and the last column in $A$. If $k>0$, then

$$
\begin{aligned}
T_{k}^{\prime}=A_{2, k+1}+A_{3, k+2}+\cdots+ & A_{n-k, n-1} \\
& =T_{k-1}-A_{1, k}-A_{n-k+1, n}=T_{k-1}-A_{1, k}+1 \geq T_{k-1} .
\end{aligned}
$$

If $k \leq 0$, then $T_{k}^{\prime}=T_{k-1}$. Hence for every $k, T_{k}^{\prime} \geq T_{k-1}$. It follows that $\bar{H}^{\prime} \geq \bar{H}+1$, $\underline{H}^{\prime} \geq \underline{H}+1$, and hence that $H_{\text {Greedy }}^{\prime} \geq H_{\text {Greedy }}+1$. West can therefore take at least

$$
\left\lceil\frac{n-1+H_{\text {Greedy }}^{\prime}}{2}\right\rceil \geq\left\lceil\frac{n+H_{\text {Greedy }}}{2}\right\rceil
$$

tricks, which was to be proved.

Case 3: East is on lead, and plays a card that West can beat. Suppose that East leads the card $E_{j}$, and that West takes the trick with the card $W_{i}$ such that $i$ is minimal with $W_{i}>E_{j}$. We can assume without loss of generality that $E_{j}$ is the highest card in its sequence. We have to show that West can take at least

$$
\left\lceil\frac{n+H_{\text {Greedy }}}{2}\right\rceil
$$

tricks. By induction, he can take

$$
1+\left\lfloor\frac{n-1+H_{\text {Greedy }}^{\prime}}{2}\right\rfloor=\left\lceil\frac{n+H_{\text {Greedy }}^{\prime}}{2}\right\rceil
$$

tricks. It would therefore be enough to show that $H_{\text {Greedy }}^{\prime} \geq H_{\text {Greedy }}$. However, this need not always be the case. In the following, we therefore assume that $H_{\text {Greedy }}^{\prime}<H_{\text {Greedy }}$. We 
show that this implies that $H_{\text {Greedy }}^{\prime}=H_{\text {Greedy }}-1$, and that $H_{\text {Greedy }} \equiv n(\bmod 2)$. This in turn implies that (13) equals

$$
\left\lceil\frac{n+H_{\text {Greedy }}-1}{2}\right\rceil=\left\lfloor\frac{n+H_{\text {Greedy }}}{2}\right\rfloor=\frac{n+H_{\text {Greedy }}}{2}=\left\lceil\frac{n+H_{\text {Greedy }}}{2}\right\rceil .
$$

We will use the fact that for any $k, T_{k} \equiv n+k(\bmod 2)$, and similarly, $T_{k}^{\prime} \equiv n-1+k$ $(\bmod 2)$.

By Lemma 5.1,

$$
T_{k}^{\prime}= \begin{cases}T_{k}+1, & \text { if } k>j-i \\ T_{k}-1, & \text { if } k \leq j-i .\end{cases}
$$

In particular, for any $k$, if $T_{k}>0$, then $T_{k}^{\prime} \geq 0$. Suppose that $T_{\underline{H}}>0$. Then $T_{\underline{H}}^{\prime} \geq 0$, and $H_{\text {Greedy }}^{\prime} \geq \underline{H}$. The only way we can have $H_{\text {Greedy }}^{\prime}<H_{\text {Greedy }}$ is therefore that $H_{\text {Greedy }}=$ $\bar{H}=\underline{H}+1$ and $H_{\text {Greedy }}^{\prime}=\underline{H}$. In this case $T_{\underline{H}}^{\prime}=0$. Hence $\underline{H} \equiv n-1(\bmod 2)$, and $H_{\text {Greedy }}=\bar{H} \equiv n(\bmod 2)$, as was desired.

If on the other hand $T_{\underline{H}}=0$, then clearly $\bar{H}=\underline{H}=H_{\text {Greedy }} \equiv n(\bmod 2)$. By Lemma $5.2, T_{H_{\text {Greedy }}-1}>0$. By Lemma 5.1, $T_{H_{\text {Greed }}-1}^{\prime} \geq 0$, so that $H_{\text {Greedy }}^{\prime} \geq H_{\text {Greedy }}-1$.

Theorem 6.1 shows that Greedy Whist has a certain symmetry property that Whist is lacking. Interchanging the East and West hands and reversing the order of the cards does not change the game theoretical value.

If $n$ is odd, then $T_{0} \neq 0$, and hence $H_{\text {Greedy }} \neq 0$. This implies the following:

Theorem 6.2. In greedy whist, if $n$ is odd, then the same player will take the majority of the tricks regardless of the location of the lead. In other words, if $n=2 k+1$, then it is impossible to arrange the cards so that each of the players is able to take $k+1$ tricks if the other is on lead.

\section{A generalization of Greedy Whist}

It would be possible to give a direct proof that the normal strategy is optimal also for Five-card. However, we prefer to prove this in a more general framework that provides an explanation for the fact that the same strategy is optimal for two seemingly different games.

Let $L_{n}$ be the lattice of all subsets of $\{1, \ldots, n\}$, ordered by the following relation: Let $x=\left\{x_{1}, \ldots, x_{p}\right\}$, and $y=\left\{y_{1}, \ldots, y_{q}\right\}$ be subsets of $\{1, \ldots, n\}$, where $x_{1}<\cdots<x_{p}$ and $y_{1}<\cdots<y_{q}$. Then $x \leq y$ in $L_{n}$ if and only if $p \leq q$ and for $i=0, \ldots, p-1, x_{p-i} \leq y_{q-i}$. $L_{n}$ is a graded lattice, and the rank of an element of $L_{n}$ is simply the sum of its elements. If $x, y \in L_{n}$, then $y$ covers $x$ iff $y$ is obtained from $x$ either by inserting the element 1 (provided $1 \notin x$ ) or by replacing an element $i$ of $x$ with $i+1$ (provided $i+1 \notin x$ ).

Let $\phi: L_{n} \rightarrow \mathbb{R}$ be an order preserving function. The game of Generalized Greedy Whist is played with $n$ cards on each hand, and with the greedy rule. The outcome of the 
game is the set of all $i$ such that West won the $i$ :th trick. The score is defined to be $\phi(x)$, where $x$ is the outcome. West tries to maximize the score, and East tries to minimize it.

Note that with $\phi(x)=|x|$, this is Greedy Whist, while

$$
\phi(x)= \begin{cases}1, & \text { if } n \in x \\ 0, & \text { if } n \notin x\end{cases}
$$

defines the game of Five-card. We prove the following:

Theorem 7.1. The normal strategy is optimal in Generalized Greedy Whist.

We say that a subset of $\{1, \ldots, n\}$ is alternating if either it consists of all even or all odd numbers below a certain limit, or it is the complement of such a set. For example, the alternating subsets of $\{1,2,3,4\}$ are $\emptyset,\{1\},\{2\},\{1,3\},\{2,4\},\{1,3,4\},\{2,3,4\}$ and $\{1,2,3,4\}$.

If both players follow the normal strategy, then the outcome of the game will be an alternating set, since the lead will alternate up to the point where all the cards of the player on lead are high. The empty set and the full set $\{1, \ldots, n\}$ are alternating. For every $k$ in the interval $0<k<n$, there are two alternating sets with $k$ elements, one that contains the number 1 and one that doesn't. Two alternating sets can always be compared with respect to the ordering of $L_{n}$ in the following way: If they have different cardinality, the larger one is greater in $L_{n}$. If they have the same number of elements, then the one that doesn't contain 1 is greater. Hence there are $2 n$ alternating subsets of $\{1, \ldots, n\}$, and they form a chain in $L_{n}$.

Proof of Theorem 7.1. We now prove Theorem 7.1 by induction on $n$. We consider a game of $n$ card Generalized Greedy Whist. We assume without loss of generality that West is on lead, and that not all West's cards are high. By induction we can also assume that from the second trick on, the players will follow the normal strategy. This implies that regardless of the play in the first trick, the outcome of the game must be an alternating set, for either West will cash a high card and stay on lead, or East will win the first trick and obtain the lead, and in either case, it follows from the induction hypothesis that the outcome will be alternating.

Once we know that the outcome of the game must be an alternating set, the players will first of all try to maximize the number of tricks they take, and with the choice between different lines of play that give the same number of tricks, they prefer losing the first trick before winning it. East has no influence on whether or not he wins the first trick. East's only objective when playing second in the first trick is therefore to maximize the number of tricks he can take. Therefore by Theorem 6.1 it is optimal for East to follow the normal strategy even in the first trick.

By Theorem 6.1, a normal lead from West maximizes the number of tricks West takes, given that both players follow the normal strategy after the first lead. At the same time, it avoids winning the first trick. Therefore, it is optimal for West to make a normal lead. This completes the induction. 
This shows that with the greedy rule, there is never a conflict between trying to take as many tricks as possible, and trying to take them as late as possible. Without the greedy rule, this is no longer true, as the following example shows:

$$
\begin{array}{ll}
\text { West : } & \text { East : } \\
\text { K Q } 87 & \text { A J } 109
\end{array}
$$

East, who has the ace, can of course take the last trick if he wants to. Without the greedy rule, there is no way to force him to play the ace before the last trick. With West on lead, it is also possible for East to take three of the four tricks against any lead. However, if West leads the king, it is impossible for East to accomplish these two tasks simultaneously. To keep West from scoring both his king and his queen, East must win the first trick with the ace, thereby giving West the opportunity to take the last trick with the queen. Hence without the greedy rule, the analogue of Theorem 7.1 is false.

\section{$7.1 \quad$ Five-card}

The following theorem determines the game theoretical value of Five-card:

Theorem 7.2. If $\operatorname{tr}(A)>0$, then West has a winning strategy at Five-card. If $\operatorname{tr}(A)<0$, then East has a winning strategy. Finally, if $\operatorname{tr}(A)=0$, then the player on lead has a winning strategy.

Proof. Note that $\operatorname{tr}(A)=T_{0}$. We already know that the normal strategy is optimal. We therefore assume that both players follow the normal strategy. We prove the theorem by induction on $n$. Suppose without loss of generality that West is on lead, and that not all of West's cards are high. West will lead a card $W_{i}$, and East will take the trick with the card $E_{j}$ so that $A_{i, j}=-1$, with $j-i$ as small as possible. By Theorem 5.1,

$$
T_{0}^{\prime}= \begin{cases}T_{0}+1, & \text { if } j-i \leq 0 \\ T_{0}-1, & \text { if } j-i>0\end{cases}
$$

We have to show that if $T_{0} \geq 0$, then West has a winning strategy, while if $T_{0}<0$, East has a winning strategy. If $T_{0}<0$, then $T_{0}^{\prime} \leq 0$, with East on lead after the first trick. Hence by induction, East has a winning strategy. If $T_{0} \geq 0$, then if $j-i \leq 0$, we have $T_{0}^{\prime}>0$. If on the other hand $j-i>0$, then by the choice of $i$ and $j$, we must have $n \geq 2$ and $T_{0}=n$, from which it follows that $T_{0}^{\prime}=n-1>0$. Hence in either case, $T_{0}^{\prime}>0$. By induction, West has a winning strategy.

\section{The normal strategy in Whist}

In this section, we apply the normal strategy to the game of (relaxed) Whist. Since we know from Example (5) that the normal strategy is not optimal, this may seem like a strange thing to do, but it turns out that the normal strategy performs quite well also 
in relaxed whist. We show that the game theoretical value of Whist differs from that of Greedy Whist by at most one trick. In Section 6, we saw that the number $H_{\text {Greedy }}$, giving the value of Greedy Whist, is the one of $\bar{H}$ and $\underline{H}$ which is greater in absolute value. We will show that the corresponding number $H=H_{W h i s t}$ for Whist is always either $\bar{H}$ or $\underline{H}$, but that the possibility of holding up will sometimes make $H$ differ from $H_{\text {Greedy }}$.

Theorem 8.1. For every distribution of the cards, there is a number $H$ with the property that with optimal play from both players, West will take

$$
\frac{n+H}{2}
$$

tricks, rounded up if East has the lead, and down if West has the lead. Moreover, either $H=\bar{H}$ or $H=\underline{H}$.

It follows from the first statement that it is never an advantage to have the lead, but that the advantage of not having the lead is never worth more than one trick. These facts were established by other methods in [2]. Conversely, knowing that at most one trick depends on the location of the lead, we can describe the game theoretical value of a card distribution by a single number by summing the number of tricks that West takes with and without the lead respectively. If we normalize by subtracting $n$, we get a number with the property of $H$ in Theorem 8.1. Hence the second statement is the nontrivial part of Theorem 8.1.

We can rephrase Theorem 8.1 in terms of the number of tricks that each player is able to take.

Theorem 8.2. Using the normal strategy, West will take at least

$$
\frac{n+\underline{H}}{2}
$$

tricks, and similarly, East will take at least

$$
\frac{n-\bar{H}}{2}
$$

tricks, rounded in favor of the player not on lead in the first trick.

Theorem 8.2 shows that completely ignoring the technique of holding up will never cost more than one trick compared to optimal play. Clearly Theorem 8.2 implies Theorem 8.1.

Proof of Theorem 8.2. We prove that West will take at least

$$
\frac{n+\underline{H}}{2}
$$

tricks (rounded in the usual way). The corresponding statement for East follows by interchanging the East and West hands. At the risk of repeating some of the arguments, we mimic the proof of Theorem 6.1. The only essential difference between this proof and 
the proof of Theorem 6.1 is that here we have to consider the possibility that East holds up when West leads.

Case 1: West has the lead. If all West's cards are high, he will take $n$ tricks, which was to be proved. We therefore assume that West has a card which is smaller than one of East's cards, that is, that $A_{1, n}=-1$. As usual, we choose $i$ and $j$ such that $A_{i, j}=-1$, with $j-i$ as small as possible, and let West play the card $W_{i}$. We have to show that West can take at least

$$
\left\lfloor\frac{n+\underline{H}}{2}\right\rfloor
$$

tricks.

Case 1A: East takes the trick. By induction, West will be able to take at least

$$
\left\lceil\frac{n-1+\underline{H}^{\prime}}{2}\right\rceil=\left\lfloor\frac{n+\underline{H}^{\prime}}{2}\right\rfloor
$$

tricks. Hence it suffices to show that $\underline{H}^{\prime} \geq \underline{H}$. We can now assume that East takes the trick with the card $E_{j}$, since this will minimize each entry of $A^{\prime}$, and thereby minimize $T_{k}^{\prime}$ for every $k$. By Lemma $5.1, T_{k}^{\prime}=T_{k}+1$ if $k \geq j-i$. On the other hand, if $k<j-i$, then by the choice of $i$ and $j$, all the terms contributing to $T_{k}^{\prime}$ are positive. This shows that for $-n+1<k<n-1$,

$$
T_{k}^{\prime} \geq \min \left(1, T_{k}+1\right)
$$

It follows that $\underline{H}^{\prime} \geq \underline{H}$.

Case 1B: East holds up. We have to show that West can take at least

$$
\left\lfloor\frac{n+\underline{H}}{2}\right\rfloor
$$

tricks. By induction, he can take

$$
1+\left\lfloor\frac{n-1+\underline{H}^{\prime}}{2}\right\rfloor=\left\lfloor\frac{n+1+\underline{H^{\prime}}}{2}\right\rfloor
$$

tricks. We therefore show that $\underline{H}^{\prime} \geq \underline{H}-1$.

We can assume that East plays his smallest card, since this minimizes $T_{k}^{\prime}$ for every $k$. This means that $A^{\prime}$ is obtained from $A$ by deleting the $i$ th row and the first column. If $k<j-i-1$, then all the terms in $T_{k}^{\prime}$ are positive. If $k \geq j-i-1$, and $k<0$, then

$$
\begin{aligned}
T_{k}^{\prime}=T_{k+1}-A_{-k, 1}-A_{i, k+i+1}+\sum_{m=i+1}^{n}\left(A_{m, m+k+1}-A_{m, m+k}\right) & \\
& \quad \geq T_{k+1}-A_{-k, 1}+1 \geq T_{k+1} .
\end{aligned}
$$

If $k \geq j-i-1$ and $k \geq 0$, then

$$
T_{k}^{\prime}=T_{k+1}+\sum_{l=i+k+1}^{n}\left(A_{l-k, l}-A_{l-k-1, l}\right) \geq T_{k+1} .
$$


Hence for $-n+1<k<n-1, T_{k}^{\prime} \geq \min \left(1, T_{k+1}\right)$. It follows that $\underline{H}^{\prime} \geq \underline{H}-1$.

Case 2: East is on lead, and leads a high card. If East leads a high card, West will play his smallest card. This means that $A^{\prime}$ is obtained by deleting the first row and the last column of $A$. If $k>0$, then

$$
\begin{aligned}
T_{k}^{\prime}=A_{2, k+1}+A_{3, k+2}+\cdots+ & A_{n-k, n-1} \\
& =T_{k-1}-A_{1, k}-A_{n-k+1, n}=T_{k-1}-A_{1, k}+1 \geq T_{k-1} .
\end{aligned}
$$

If $k \leq 0$, then $T_{k}^{\prime}=T_{k-1}$. Hence for every $k, T_{k}^{\prime} \geq T_{k-1}$. It follows that $\underline{H}^{\prime} \geq \underline{H}+1$. West can take at least

$$
\left\lceil\frac{n-1+\underline{H^{\prime}}}{2}\right\rceil \geq\left\lceil\frac{n+\underline{H}}{2}\right\rceil
$$

tricks, which was to be proved.

Case 3: East is on lead, and plays a card that West can beat. In this case, we will assume that West always takes the trick (as cheaply as possible). We have already seen that this strategy is not always optimal. However, it is sufficient for the proof of Theorem 8.2. Suppose that East leads the card $E_{j}$, and that West takes the trick with the card $W_{i}$ such that $i$ is minimal with $W_{i}>E_{j}$. We can assume without loss of generality that $E_{j}$ is the highest card in its sequence. We have to show that West can take at least

$$
\left\lceil\frac{n+\underline{H}}{2}\right\rceil
$$

tricks. By induction, he can take

$$
1+\left\lfloor\frac{n-1+\underline{H^{\prime}}}{2}\right\rfloor=\left\lceil\frac{n+\underline{H}^{\prime}}{2}\right\rceil
$$

tricks. It would therefore be enough that $\underline{H}^{\prime} \geq \underline{H}$, but this need not always be the case. In the following, we therefore assume that $\underline{H}^{\prime}<\underline{H}$. We show that this implies that $\underline{H}^{\prime}=\underline{H}-1$, and that $\underline{H} \equiv n(\bmod 2)$. This means that $(17)$ equals $(18)$ anyway. We will use the fact that for any $k, T_{k} \equiv n+k(\bmod 2)$, and similarly, $T_{k}^{\prime} \equiv n-1+k(\bmod 2)$.

By Lemma 5.1,

$$
T_{k}^{\prime}= \begin{cases}T_{k}+1, & \text { if } k>j-i \\ T_{k}-1, & \text { if } k \leq j-i .\end{cases}
$$

In particular, for any $k$, if $T_{k}>0$, then $T_{k}^{\prime} \geq 0$. If $T(\underline{H})>0$, then $T_{\underline{H}}^{\prime} \geq 0$, and consequently, $\underline{H}^{\prime} \geq \underline{H}$. If on the other hand $T_{\underline{H}}=0$, then clearly $\underline{H} \equiv n(\bmod 2)$. By Lemma 5.2, $T_{\underline{H}-1}>0$. By Lemma 5.1, $T_{\underline{H}-1}^{\prime} \geq 0$, so that $\underline{H}^{\prime} \geq \underline{H}-1$.

For a fixed $n$, if the cards are dealt randomly, and it is decided randomly which player to lead in the first trick, then with probability at least $3 / 4$, the normal strategy is optimal for West (in the weak sense that against optimal play from East, West is guaranteed at least as many tricks as with any other strategy, although the normal strategy does not necessarily take maximal advantage of mistakes from East). This follows from the next theorem: 
Theorem 8.3. Suppose we play a certain distribution of the cards four times, once with the West hand, leading to the first trick, once with West playing second in the first trick, and similarly once with the East hand leading to the first trick and once with the East hand playing second in the first trick. Then the normal strategy is guaranteed to score a total of at least $2 n-1$ tricks, compared to $2 n$ tricks with optimal play. Moreover, it will score at least $n-1$ tricks in the two games where we lead to the first trick, and at least $n$ tricks in the two games where we play second in the first trick.

Proof. We apply Theorem 8.2. We use the fact that $\underline{H}-\bar{H} \geq-1$. In the two games where we lead to the first trick, we get at least

$$
\begin{aligned}
\left\lfloor\frac{n+\underline{H}}{2}\right\rfloor+\left\lfloor\frac{n-\bar{H}}{2}\right\rfloor \geq\left\lceil\frac{n+\underline{H}}{2}-\frac{1}{2}+\frac{n-\bar{H}}{2}-\frac{1}{2}\right\rceil \\
\geq\left\lceil\frac{2 n-1}{2}-1\right\rceil=\lceil n-3 / 2\rceil=n-1
\end{aligned}
$$

tricks. Similarly, playing second, we get at least

$$
\left\lceil\frac{n+\underline{H}}{2}\right\rceil+\left\lceil\frac{n-\bar{H}}{2}\right\rceil \geq\left\lceil\frac{n+\underline{H}}{2}+\frac{n-\bar{H}}{2}\right\rceil \geq\lceil n-1 / 2\rceil=n
$$

tricks.

It follows that in at least three of these four games, the normal strategy is guaranteed to score at least the game theoretical value of the deal. It can be shown by simple strategy-stealing arguments [2] that

(a) In Whist, it is never an advantage to have the lead.

(b) The advantage of not having the lead can be worth at most one trick.

Theorem 8.3 provides a completely constructive proof of these facts, using the normal strategy.

\section{The game theoretical value of Whist}

\subsection{The main theorem}

After a little experimentation, one can formulate several conjectures about the game theoretical value of whist for certain card distributions. It turns out that for the majority of card distributions, the position of the highest card, hereafter called the ace, determines whether $H=\bar{H}$ or $\underline{H}$ (in favor of the holder of the ace). Careful study of the exceptions to this rule reveals the following theorem, which allows a somewhat tedious but still fairly straightforward proof by induction.

Theorem 9.1. The game theoretical value of Whist is given by the following: Suppose that East has the ace. If 
1.

$$
\bar{H} \geq 0
$$

2.

$$
A_{2,2+\bar{H}}+A_{3,3+\bar{H}}+\cdots+A_{n-1-\bar{H}, n-1} \geq 0
$$

and

3. there is an integer $k$ such that

$$
\sum_{i=2}^{2 k} A_{i, i+\underline{H}}>0
$$

and

$$
\sum_{i=2 k+1}^{n-\bar{H}-1} A_{i, i+\bar{H}}>0
$$

then $H=\bar{H}$. Otherwise $H=\underline{H}$.

Similarly, if West has the ace, then $H=\bar{H}$ unless

1.

$$
\underline{H} \leq 0
$$

2.

$$
A_{2-\underline{H}, 2}+A_{3-\underline{H}, 3}+\cdots+A_{n-1, n-1+\underline{H}} \leq 0,
$$

and

3. there is an integer $k$ such that

$$
\sum_{i=2}^{2 k} A_{i-\bar{H}, i}<0
$$

and

$$
\sum_{i=2 k+1}^{n+\underline{H}-1} A_{i-\underline{H}, i}<0 .
$$

\subsection{Organization of the proof}

In the proof of Theorem 9.1, we will make implicit use of Theorem 8.2. We need only distinguish between the cases where $H=\bar{H}$ and the cases where $H=\underline{H}$. Hence we can assume that $\bar{H} \neq \underline{H}$, that is, $\bar{H}=\underline{H}+1$.

If we want to prove that for a certain deal, $H=\bar{H}$, it is sufficient to give a strategy for West showing that $H \geq \bar{H}$. If $n \equiv \bar{H}(\bmod 2)$, we need only consider the case that West is on lead, since if East has the lead, the number of tricks taken with optimal play is 
independent of whether $H=\bar{H}$ or $H=\underline{H}$. Similarly, if $n \equiv \underline{H}(\bmod 2)$, we can assume that East is on lead. We divide the deals for which we claim that $H=\bar{H}$ into four classes:

I. West has the ace and $\underline{H}>0$.

II. West has the ace, $\underline{H} \leq 0$, and $A_{2-\underline{H}, 2}+\cdots+A_{n-1, n-1+\underline{H}}>0$.

III. West has the ace, $\underline{H} \leq 0, A_{2-\underline{H}, 2}+\cdots+A_{n-1, n-1+\underline{H}} \leq 0$, and there is no $k$ such that

$$
\sum_{i=2}^{2 k} A_{i-\bar{H}, i}<0
$$

and

$$
\sum_{i=2 k+1}^{n-1+\underline{H}} A_{i-\underline{H}, i}<0 .
$$

IV. East has the ace, $\bar{H} \geq 0, A_{2,2+\bar{H}}+\cdots+A_{n-1-\bar{H}, n-1} \geq 0$, and there is a number $k$ such that

$$
\sum_{i=2}^{2 k} A_{i, i+\underline{H}}>0
$$

and

$$
\sum_{i=2 k+1}^{n-1-\bar{H}} A_{i, i+\bar{H}}>0 .
$$

We prove by induction on $n$ that for each of these four cases, $H=\bar{H}$. We first establish two lemmas.

Lemma 9.2. If there is a number $k$ such that

$$
\sum_{i=2}^{2 k} A_{i, i+\underline{H}}
$$

and

$$
\sum_{i=2 k+1}^{n-\bar{H}-1} A_{i, i+\bar{H}}
$$

are positive, then the largest value of $k$ which makes (21) positive will also make (20) positive.

Proof. Let

$$
S(k)=\sum_{i=2}^{2 k} A_{i, i+\underline{H}}+\sum_{i=2 k+1}^{n-\bar{H}-1} A_{i, i+\bar{H}} .
$$

Then

$$
S(k+1)-S(k)=A_{2 k+1,2 k+1+\underline{H}}-A_{2 k+1,2 k+1+\bar{H}}+A_{2 k+2,2 k+2+\underline{H}}-A_{2 k+2,2 k+2+\bar{H}} \geq 0 .
$$


Hence $S(k)$ is increasing with $k$. If $k$ is maximal such that (21) is positive, then (21) is equal to 1 or 2 depending on whether $n \equiv \bar{H}(\bmod 2)$ or not. If for this value of $k,(20)$ is not positive, then since $(20)$ is odd, $S(k) \leq 1$. Therefore no smaller value of $k$ can make both (20) and (21) positive.

Hence when considering case IV, we may assume that $k$ is maximal such that the second sum is positive. The following lemma allows us to exclude some cases.

Lemma 9.3. There is no point in holding up if the opponent leads his smallest card.

In other words, if the opponent plays his smallest card, taking the trick as cheaply as possible is at least as good as holding up.

Proof. Suppose the sequence containing the opponent's smallest card contains $m$ cards. If $m=1$, there is no point in holding up, since the resulting $(n-1)$-card deal will be equivalent to that resulting from taking the trick. If we take, we get the lead, but we know from Theorem 8.3 that having the lead will cost at most one trick.

For every strategy, there is a number $k$ such that if the opponent plays his cards from the bottom up, we will hold up $k$ times and take trick $k+1$. We can assume that $0 \leq k<m$. We claim that if $k \geq 1$, then it is at least as good to hold up only $k-1$ times, take the $k$ th trick, and then lead the smallest remaining card. When we lead the smallest card in trick $k+1$, the opponent has no smaller card, and will therefore take the trick with his smallest card. The two lines of play therefore lead to the same position, with the same number of tricks for both players, but in the latter case with the opponent on lead. Hence holding up only $k-1$ times is at least as good as holding up $k$ times. It follows that taking the first trick is at least as good as anything else.

\subsection{Proof of Theorem 9.1}

We now turn to the proof of Theorem 9.1. We consider a positive integer $n$ and a deal $D$ with $n$ cards on each hand, and assume that the theorem holds for all $(n-1)$-card deals. We consider cases I-IV as described above, and in each of these four cases, we consider first the case that $n \equiv \underline{H}(\bmod 2)$ and East has the lead, and then the case that $n \equiv \bar{H}$ (mod 2) with West on lead. This gives us eight cases, and several of these are further divided into subcases. The eight main cases are labeled $I(E), I(W), \ldots, I V(E), I V(W)$. In each case, we begin by describing an optimal play for West. This means that the proof of Theorem 9.1 also gives an explicit optimal strategy for the game.

We do not repeat all the calculations from the proofs of Theorems 6.1 and 8.2. If the player who is not on lead takes the first trick, then we have to show that $H^{\prime} \geq \bar{H}$. If West holds up, we show that $H^{\prime} \geq \bar{H}+1$, while if East holds up, it suffices to show that $H^{\prime} \geq \bar{H}-1$.

Case $I(E)$. We first consider hands of type I with $n \equiv \underline{H}(\bmod 2)$, and East on lead. West takes the trick if he can do so with a card smaller than the ace. Otherwise he holds up.

THE ELECTRONic Journal of COMBinatorics 12 (2005), \#R43 
Suppose first that West can take the trick and still hold the ace. Since $n \equiv \underline{H}, T_{\underline{H}}$ is even, and therefore at least 2. By Lemma 5.1, $T_{\underline{H}}^{\prime} \leq 1$. Therefore, $\underline{H}^{\prime} \geq \underline{H}>0$. It follows that $D^{\prime}$ belongs to case I. By induction, $H^{\prime}={\overline{H^{\prime}}}^{\prime} \geq \bar{H}$.

Secondly, suppose that East leads a card that West can beat only with the ace. West holds up. In this case, $A^{\prime}$ is obtained by deleting the first row and the last column of $A$. Since $A_{n-\underline{H}, n}=-1$, we have

$$
\begin{aligned}
T_{\underline{H}+1}^{\prime}=A_{2,2+\underline{H}}+\cdots+A_{n-\underline{H}-1, n-1}=T_{\underline{H}}-A_{1,1+\underline{H}} & -A_{n-\underline{H}, n} \\
& =T_{\underline{H}}-A_{1,1+\underline{H}}+1 \geq T_{\underline{H}}>0 .
\end{aligned}
$$

Hence $\bar{H}^{\prime} \geq \bar{H}+1$. Again $D^{\prime}$ belongs to Case I, so that $H^{\prime} \geq \bar{H}+1$.

Case $I(W)$. We now consider the case that $n \equiv \bar{H}(\bmod 2)$ with West on lead. If there is a negative entry on the $\underline{H}$-diagonal of $A$, that is, on the diagonal $\left\{A_{i, j}: j-i=\underline{H}\right\}$, then West leads the smallest card corresponding to a row with such an entry. If there is no negative entry on the $\underline{H}$-diagonal, West leads his smallest card.

Consider first the possibility that East takes the trick. By Lemma 5.1, we have

$$
T_{\underline{H}}^{\prime}=T_{\underline{H}}-A_{i, i+\underline{H}} .
$$

Either $A_{i, i+\underline{H}}$ is negative, which implies that $T_{\underline{H}}^{\prime}>T_{\underline{H}}>0$, so that $\bar{H}^{\prime} \geq \bar{H}$, or the $\underline{H}$-diagonal of $A$ consists only of positive entries. In the latter case, we must have $T_{\underline{H}}^{\prime}>0$ anyway, since $\underline{H}=n-1$ is impossible. Therefore $D^{\prime}$ belongs to case I with $H^{\prime} \geq \bar{H}$.

Suppose on the other hand that East holds up. By Lemma 9.3, we can assume that $i>1$, so that $A_{i, i+\underline{H}}=-1$. We have

$$
T_{\underline{H}-1}^{\prime}=A_{1,1+\underline{H}}+\cdots+A_{i-1, i-1+\underline{H}}+A_{i+1, i+\underline{H}}+\cdots+A_{n+1-\underline{H}, n} \geq T_{\underline{H}}>0,
$$

so that $\underline{H}^{\prime} \geq \underline{H}-1$. If $\underline{H}>1$, this is already sufficient, since $D^{\prime}$ will belong to case I. Moreover, if $\underline{H}=1$, we have

$$
\begin{aligned}
A_{2,2}^{\prime}+\cdots+A_{n-2, n-2}^{\prime}=A_{2,3}+\cdots & +A_{i-1, i}+A_{i+1, i+1}+\cdots+A_{n-1, n-1} \\
\geq A_{2,3}+\cdots+ & A_{i-1, i}+A_{i+1, i+2}+\cdots+A_{n-1, n} \\
& =T_{1}-A_{1,2}-A_{i, i+1}=T_{1}-A_{1,2}+1 \geq T_{1}>0 .
\end{aligned}
$$

In this case, $D^{\prime}$ belongs to Case II, and $H^{\prime} \geq 1$.

Case $I I(E)$. We now consider hands of type II, with $n \equiv \underline{H}(\bmod 2)$ and East on lead. As in Case I, West takes the trick provided he can do so with a card smaller than the ace. Otherwise he holds up.

Suppose that West can take the trick with a card smaller than the ace. Since

$$
A_{2-\underline{H}, 2}+\cdots+A_{n-1, n-1+\underline{H}}
$$

is even, it is at least 2. By Lemma 5.1,

$$
A_{2-\underline{H}, 2}^{\prime}+\cdots+A_{n-2, n-2+\underline{H}}^{\prime} \geq A_{2-\underline{H}, 2}+\cdots+A_{n-1, n-1+\underline{H}}-1>0 .
$$


Therefore $D^{\prime}$ belongs to case II, and by induction, $H^{\prime} \geq \bar{H}$.

Suppose now that East leads a card that West can beat only with the ace. West holds up, and $A^{\prime}$ is obtained by deleting the first row and the last column of $A$. If $\underline{H}=0$, then

$$
T_{1}^{\prime}=A_{2-\underline{H}, 2}+\cdots+A_{n-1, n-1+\underline{H}}>0,
$$

so that $\bar{H}^{\prime} \geq 2$, with $D^{\prime}$ belonging to case I.

If $\underline{H}<0$, then $T_{\underline{H}+1}^{\prime}=T_{\underline{H}}$. We have $\bar{H}^{\prime}=\bar{H}+1$, and

$$
A_{2-\underline{H^{\prime}, 2}}^{\prime}+\cdots+A_{n-2, n-2+\underline{H}^{\prime}}^{\prime}=A_{2-\underline{H}, 2}+\cdots+A_{n-1, n-1+\underline{H}}>0 .
$$

In this case $D^{\prime}$ belongs to case II, and by induction $H^{\prime}=\bar{H}^{\prime} \geq \bar{H}+1$.

Case $I I(W)$. Suppose now that in case II, $n \equiv \bar{H}(\bmod 2)$, and that West is on lead. West leads the smallest card $W_{i}$ such that $i \geq 2-\underline{H}$ and $A_{i, i+\underline{H}}=-1$, that is, West leads the smallest card corresponding to a negative entry on the $\underline{H}$-diagonal of $A$, except if this entry is in the first column. If there is no such $i$, West leads his smallest card.

Consider the possibility that East takes the trick. If all the entries on the $\underline{H}$-diagonal in $A$ are positive, then we have $T_{\underline{H}}^{\prime}=T_{\underline{H}}-1=n-1+\underline{H}>0$, and $A_{2-\underline{H^{\prime}, 2}}^{\prime}+\cdots+A_{n-2, n-2+\underline{H}^{\prime}}^{\prime}=$ $n-3+\underline{H}$. Since the sum

$$
A_{2-\underline{H}, 2}+\cdots+A_{n-1, n-1+\underline{H}}
$$

contains at least one term, $\underline{H}$ is at least $-n+3$. If $\underline{H}>-n+3$, then $A_{2-\underline{H}^{\prime}, 2}^{\prime}+\cdots+$ $A_{n-2, n-2+H^{\prime}}^{\prime}>0$, and $D^{\prime}$ belongs to case II again. If equality holds, then $D^{\prime}$ reduces to a rather trivial special case of III, where there is not even a value of $k$ such that both sums involved have at least one term.

If there is a negative entry on the $\underline{H}$-diagonal which is not in the first row, then by Lemma 5.1, we have $T_{\underline{H}}^{\prime}=T_{\underline{H}}-A_{i, i+\underline{H}}=T_{\underline{H}}+1>0$, and similarly,

$$
A_{2-\underline{H}, 2}^{\prime}+\cdots+A_{n-2, n-2+\underline{H}}^{\prime}=A_{2-\underline{H}, 2}+\cdots+A_{n-1, n-1+\underline{H}}+1>0 .
$$

This implies that $\bar{H}^{\prime} \geq \bar{H}$, and by case II of the induction hypothesis, $H^{\prime}=\bar{H}^{\prime}$.

Suppose on the other hand that East holds up. Then by Lemma 9.3, we can assume that West did not play his smallest card. Hence $A^{\prime}$ is obtained from $A$ by deleting the first column, and the row $i$ in which the entry on the $\underline{H}$-diagonal is negative. We have

$$
\begin{aligned}
& T_{\underline{H}-1}^{\prime}=A_{2-\underline{H}, 2}+\cdots+A_{i-1, i-1+\underline{H}}+A_{i+1, i+\underline{H}}+\cdots+A_{n, n+\underline{H}} \\
& \geq A_{2-\underline{H}, 2}+\cdots+A_{i-1, i-1+\underline{H}}+A_{i+1, i+1+\underline{H}}+\cdots+A_{n-1, n-1+\underline{H}}+1 \\
& \quad=A_{2-\underline{H}, 2}+\cdots+A_{n-1, n-1+\underline{H}}-A_{i, i+\underline{H}}+1 \\
& \quad=A_{2-\underline{H}, 2}+\cdots+A_{n-1, n-1+\underline{H}}+2 \geq 3 .
\end{aligned}
$$

It follows that $A_{2-\underline{H}+1,2}^{\prime}+\cdots+A_{n-2, n-2+\underline{H}-1}^{\prime}>0$, and again by case II of the induction hypothesis, $H^{\prime}=\bar{H}^{\prime} \geq \bar{H}-1$. This completes the analysis of Case II.

Case $I I I(E)$. We now consider case III, with $n \equiv \underline{H}(\bmod 2)$, and East on lead. West always takes the trick if this can be done with a card smaller than the ace. If East leads 
a card that West can beat only with the ace, then West holds up if $\underline{H}<0$, but takes the trick if $\underline{H}=0$.

Suppose first that East plays a card that West can beat with a card smaller than the ace. $T_{\underline{H}}$ is even, hence at least 2 . It follows that $T_{\underline{H}}^{\prime} \geq T_{\underline{H}}-1>0$, so that $\bar{H}^{\prime} \geq \bar{H}$. We show that there is no $k$ such that

$$
\sum_{i=2}^{2 k} A_{i-\bar{H}, i}^{\prime}<0
$$

and

$$
\sum_{i=2 k+1}^{n-2+\underline{H}} A_{i-\underline{H}, i}^{\prime}<0 .
$$

Suppose for a contradiction that there is a $k$ such that these sums are negative. By Lemma 9.2, we need only consider the largest $k$ which makes the second sum negative. This means that the first term of the second sum, $A_{2 k+1-\underline{H}, 2 k+1}^{\prime}$, is negative. Suppose that East played the card $E_{j}$ in the first trick. If $j>2 k$, then

$$
\sum_{i=2}^{2 k} A_{i-\bar{H}, i}=\sum_{i=2}^{2 k} A_{i-\bar{H}, i}^{\prime}<0
$$

and

$$
\sum_{i=2 k+1}^{n-1+\underline{H}} A_{i-\underline{H}, i}=\sum_{i=2 k+1}^{n-2+\underline{H}} A_{i-\underline{H}, i}^{\prime}+A_{j-\underline{H}, j} \leq-1,
$$

a contradiction.

If on the other hand $j \leq 2 k$, then

$$
\sum_{i=2}^{2(k+1)} A_{i-\bar{H}, i}=\sum_{i=2}^{2 k} A_{i-\bar{H}, i}^{\prime}+A_{2 k+1-\underline{H}, 2 k+1}^{\prime}+A_{j-\bar{H}, j}<0,
$$

since $A_{2 k+1-\underline{H}, 2 k+1}^{\prime}=-1$, and

$$
\sum_{i=2(k+1)+1}^{n-1-\underline{H}} A_{i-\underline{H}, i}=\sum_{i=2 k+1}^{n-2-\underline{H}} A_{i-\underline{H}, i}^{\prime}+1<0 .
$$

This gives the same contradiction, now with the number $k+1$ in the role of $k$. By Case III of the induction hypothesis, $H^{\prime}=\bar{H}^{\prime} \geq \bar{H}$.

Suppose instead that East leads a card that West can beat only with the ace. We first look at the case $\underline{H}<0$. In this case, West holds up. We get $A^{\prime}$ by deleting the first row and the last column of $A$. We have

$$
T_{\underline{H}+1}^{\prime}=T_{\underline{H}}>0 \text {. }
$$


Hence $\underline{H}^{\prime} \geq \underline{H}+1$ and $\bar{H}^{\prime} \geq \bar{H}+1$. West still has the ace. Since $A_{i, j}^{\prime}=A_{i+1, j}$ whenever $j-i=\underline{H}$ or $j-i=\bar{H}$, we have, for every $k$,

$$
\sum_{i=2}^{2 k} A_{i-\bar{H}-1, i}^{\prime}=\sum_{i=2}^{2 k} A_{i-\bar{H}, i}
$$

and

$$
\sum_{i=2 k+1}^{n-1+\underline{H}} A_{i-\underline{H}-1, i}^{\prime}=\sum_{i=2 k+1}^{n-1+\underline{H}} A_{i-\underline{H}, i}
$$

By assumption, both sums cannot be negative. This case therefore reduces to Case III of the induction hypothesis.

Now we consider the case that East leads a card that West can beat only with the ace, and that $\underline{H}=0$. West takes the trick with the ace. This means that $A^{\prime}$ is obtained by deleting the last row and the last column of $A$. Since $n \equiv \underline{H}(\bmod 2), n$ is even, and so is $T_{0}$. Hence $T_{0} \geq 2$. We have $T_{0}^{\prime}=T_{0}-1>0$. Hence $\underline{H^{\prime}} \geq 0$, and $\bar{H}^{\prime} \geq 1$. Since West cannot take the first trick with a smaller card than the ace, East must have the next highest card, the "king". But we can actually assume that East has the two cards immediately below the ace, the "king" and the "queen". Indeed, if West has the ace and the queen, and East leads the king to West's ace, then the position becomes equivalent to that which would occur if East played his next highest card, which West would then take with a card equivalent to the queen. But this situation has already been analyzed. Hence we assume that East has both the "king" and the "queen", and in particular that $A_{n-1, n-1}=-1$.

Since $A_{n-1, n-1}=-1$, we must have

$$
A_{1,2}+\cdots+A_{n-3, n-2} \geq 0 .
$$

Otherwise with $k=n / 2-1$, we would have

$$
\sum_{i=2}^{2 k} A_{i-\bar{H}, i}<0
$$

and

$$
\sum_{i=2 k+1}^{n-1+\underline{H}} A_{i-\underline{H}, i}<0 .
$$

Hence

$$
T_{1}^{\prime}=A_{1,2}^{\prime}+\cdots+A_{n-2, n-1}^{\prime}=A_{1,2}+\cdots+A_{n-2, n-1} \geq A_{n-2, n-1} \geq-1 .
$$

But the left hand side of (26) is even, hence nonnegative. It follows that $\underline{H}^{\prime} \geq 1$, and that $H^{\prime} \geq \bar{H}$. This concludes Case III(E). 
Case $I I I(W)$. We now consider hands of type III with $n \equiv \bar{H}(\bmod 2)$ and West on lead. Since $A_{2-\underline{H}, 2}+\cdots+A_{n-1, n-1+\underline{H}} \leq 0$, there must be an $m$ in the interval $2-\underline{H} \leq m \leq n-1$ such that $A_{m, m+\underline{H}}=-1$. West leads the card $W_{m}$ where $m$ is maximal with this property.

Suppose first that East holds up. Then $A^{\prime}$ is obtained by deleting the $m$ :th row and the first column of $A$. We have

$$
\begin{aligned}
T_{\underline{H}-1}^{\prime}=A_{2-\underline{H}, 2}+\cdots+ & A_{m-1, m-1+\underline{H}}+A_{m+1, m+\underline{H}}+\cdots+A_{n, n-1+\underline{H}} \\
\geq A_{2-\underline{H}, 2}+ & \cdots+A_{m-1, m-1+\underline{H}}+A_{m+1, m+1+\underline{H}}+\cdots+A_{n, n+\underline{H}} \\
& =T_{\underline{H}}-A_{1+\underline{H}, 1}-A_{m+\underline{H}, m}=T_{\underline{H}}-A_{1+\underline{H}, 1}+1 \geq T_{\underline{H}}>0 .
\end{aligned}
$$

Suppose for a contradiction that $H^{\prime}<\bar{H}-1$. Since West still has the ace, there must be a $k$ such that

$$
\sum_{i=2}^{2 k} A_{i-\bar{H}+1, i}^{\prime}<0
$$

and

$$
\sum_{i=2 k+1}^{n-2+\underline{H}-1} A_{i-\underline{H}+1, i}^{\prime}<0 .
$$

By Lemma 9.2, we can assume that $k$ is the maximal number such that the second sum is negative. By the choice of $m$, the second sum must start with a matrix element above the $m$ :th row. Since $k$ is chosen maximal, we have $A_{2 k+2-\underline{H}, 2 k+1}^{\prime}=-1$. It follows that

$$
A_{2 k+2-\bar{H}, 2 k+1}^{\prime}=-1 \text {. }
$$

We have

$$
\begin{aligned}
& \sum_{i=2}^{2(k+1)} A_{i-\bar{H}, i}=\sum_{i=2}^{2(k+1)} A_{i-\bar{H}, i-1}^{\prime}=\sum_{i=1}^{2(k+1)-1} A_{i-\bar{H}+1, i}^{\prime} \\
& =\sum_{i=2}^{2 k} A_{i-\bar{H}+1, i}^{\prime}+A_{2-\bar{H}, 1}^{\prime}+A_{2 k+2-\bar{H}, 2 k+1}^{\prime} \leq \sum_{i=2}^{2 k} A_{i-\bar{H}+1, i}^{\prime}<0,
\end{aligned}
$$

and

$$
\begin{aligned}
& \sum_{i=2(k+1)+1}^{n-1+\underline{H}} A_{i-\underline{H}, i}=A_{2 k+3-\underline{H}, 2 k+2}^{\prime}+\cdots+A_{m-1, m-2+\underline{H}}^{\prime}-1+ \\
&+A_{m, m+\underline{H}}^{\prime}+\cdots+A_{n-2, n-2+\underline{H}}^{\prime} \leq A_{2 k+3-\underline{H}}^{\prime}+\cdots+A_{m-1, m-2+\underline{H}}^{\prime}+ \\
&+A_{m, m-1+\underline{H}}^{\prime}+\cdots+A_{n-2, n-3+\underline{H}}^{\prime}-1 \\
&=\sum_{i=2 k+2}^{n-3+\underline{H}} A_{i-\underline{H}+1, i}^{\prime}-1 \leq \sum_{i=2 k+1}^{n-2+\underline{H}-1} A_{i-\underline{H}+1, i}^{\prime}<0,
\end{aligned}
$$


contrary to assumption. Hence $H^{\prime} \geq \bar{H}-1$.

Suppose on the other hand that East takes the trick. Then

$$
T_{\underline{H}}^{\prime}=T_{\underline{H}}-A_{m, m+\underline{H}}=T_{\underline{H}}+1>0 .
$$

Hence $\bar{H}^{\prime} \geq \bar{H}$. Suppose that $H^{\prime} \neq \bar{H}^{\prime}$. Then there must be a $k$ such that

$$
\sum_{i=2}^{2 k} A_{i-\bar{H}, i}^{\prime}<0
$$

and

$$
\sum_{i=2 k+1}^{n-2+\underline{H}} A_{i-\underline{H}, i}^{\prime}<0
$$

By the choice of $m$, whenever $i-\underline{H} \geq m$, we have

$$
A_{i-\underline{H}, i}^{\prime}=A_{i-\underline{H}+1, i+1}=1 \text {. }
$$

In order for the sum

$$
\sum_{i=2 k+1}^{n-2+\underline{H}} A_{i-\underline{H}, i}^{\prime}
$$

to contain a negative term, it must start with an element above the $m$ th row, that is, we must have $2 k+1-\underline{H}<m$. Hence

$$
\sum_{i=2}^{2 k} A_{i-\bar{H}, i}=\sum_{i=2}^{2 k} A_{i-\bar{H}, i}^{\prime}<0
$$

and

$$
\sum_{i=2 k+1}^{n-1+\underline{H}} A_{i-\underline{H}, i}=\sum_{i=2 k+1}^{n-2+\underline{H}} A_{i-\underline{H}, i}^{\prime}+A_{m, m+\underline{H}}=\sum_{i=2 k+1}^{n-2+\underline{H}} A_{i-\underline{H}, i}^{\prime}-1<0,
$$

contrary to assumption. Hence $H^{\prime}=\bar{H}^{\prime} \geq \bar{H}$. This concludes Case III.

Case IV $(E)$. We now consider Case IV, with $n \equiv \underline{H}(\bmod 2)$, and East on lead. West will always take the trick if he can, but we first consider the case that East leads a high card. In this case, $A^{\prime}$ is obtained by deleting the first row and the last column of $A$. Then

$$
T_{\bar{H}+1}^{\prime}=A_{2,2+\bar{H}}+\cdots+A_{n-1-\bar{H}, n-1} \geq 0,
$$

so that $\underline{H^{\prime}} \geq \bar{H}+1$. This takes care of the possibility that East leads a high card. Suppose therefore that East leads a card that West can beat, and that West takes the trick as cheaply as possible. We have $T_{\underline{H}} \geq 2$, and consequently $T_{\underline{H}}^{\prime} \geq 1$. Hence $\underline{H}^{\prime} \geq \underline{H}$ and $\bar{H}^{\prime} \geq \bar{H}$.

$$
A_{2,2+\bar{H}}+\cdots+A_{n-1-\bar{H}, n-1} \geq 1
$$


since the left hand side is odd. Hence

$$
A_{2,2+\bar{H}}^{\prime}+\cdots+A_{n-2-\bar{H}, n-2}^{\prime} \geq 1-1=0 .
$$

Suppose that $k$ is maximal with

$$
\sum_{i=2 k+1}^{n-1-\bar{H}} A_{i, i+\bar{H}}>0 .
$$

Then $A_{2 k+1,2 k+1+\bar{H}}=1$. Suppose West plays the card $W_{m}$ in the first trick. The same value of $k$ will do again: If $m \geq 2 k+1$, then

$$
\sum_{i=2}^{2 k} A_{i, i+\underline{H}}^{\prime}=\sum_{i=2}^{2 k} A_{i, i+\underline{H}}>0,
$$

while if $m \leq 2 k$, then

$$
\begin{aligned}
\sum_{i=2}^{2 k} A_{i, i+\underline{H}}^{\prime}=\sum_{i=2}^{2 k} A_{i, i+\underline{H}}-A_{m, m+\underline{H}}+A_{2 k+1,2 k+1+\bar{H}} & \\
& \geq \sum_{i=2}^{2 k} A_{i, i+\underline{H}}-1+A_{2 k+1,2 k+1+\bar{H}}=\sum_{i=2}^{2 k} A_{i, i+\underline{H}}>0 .
\end{aligned}
$$

In any case, we have

$$
\sum_{i=2 k+1}^{n-2-\bar{H}} A_{i, i+\bar{H}}^{\prime} \geq \sum_{i=2 k+1}^{n-1-\bar{H}} A_{i, i+\bar{H}}-1 .
$$

The sum in the right hand side is positive. Moreover, it is even. Hence the left hand side is positive. This disposes of the case that East has the lead.

Case $I V(W)$. We now consider the case that $n \equiv \bar{H}(\bmod 2)$, and West has the lead. The strategy is simple, West will lead his highest card. Suppose first that East holds up. If $\bar{H}=0$, then

$$
T_{0}^{\prime}=A_{2,2}+\cdots+A_{n-1, n-1} \geq 0 .
$$

Hence $\underline{H}^{\prime} \geq 0$. If $\bar{H}>0$, then either $\bar{H}=1$, and

$$
T_{-1}^{\prime}=T_{0}-A_{1,1}-A_{n, n}=T_{0}-A_{1,1}+1 \geq T_{0}>0
$$

or $\bar{H}>1$ and $T_{\underline{H}-1}^{\prime}=T_{\underline{H}}>0$. In any case, $\underline{H}^{\prime} \geq \underline{H}-1$ and $\bar{H}^{\prime} \geq \bar{H}-1$. Moreover, the same value of $k$ will do:

$$
\sum_{i=2}^{2 k} A_{i, i+\underline{H}-1}^{\prime}=\sum_{i=2}^{2 k} A_{i, i+\underline{H}}>0,
$$


and

$$
\sum_{i=2 k+1}^{n-2-(\bar{H}+1)} A_{i, i+\bar{H}-1}^{\prime}=\sum_{i=2 k+1}^{n-1-\bar{H}} A_{i, i+\bar{H}}>0 .
$$

Suppose therefore that East takes the trick with a card equivalent to the ace. Then $A^{\prime}$ is obtained by deleting the last row and the last column of $A$.

Suppose first that $\underline{H} \geq 0$. Then

$$
T_{\underline{H}}^{\prime}=T_{\underline{H}}-A_{n-\underline{H}, n}=T_{\underline{H}}+1>0 .
$$

Hence $\underline{H^{\prime}} \geq \underline{H}$ and $\bar{H}^{\prime} \geq \bar{H}$. If East has the highest remaining card, then in particular $A_{n-1-\bar{H}, n-1}=-1$, and

$$
\begin{aligned}
A_{2,2+\bar{H}}^{\prime}+\cdots+A_{n-2-\bar{H}, n-2}^{\prime} & =A_{2,2+\bar{H}}+\cdots+A_{n-2-\bar{H}, n-2} \\
=A_{2,2+\bar{H}}^{\prime}+\cdots+A_{n-1-\bar{H}, n-1}^{\prime}- & A_{n-1-\bar{H}, n-1}^{\prime} \\
& \geq 0-A_{n-1-\bar{H}, n-1}=1 \geq 0 .
\end{aligned}
$$

The same value of $k$ will do:

$$
\sum_{i=2}^{2 k} A_{i, i+\underline{H}}^{\prime}=\sum_{i=2}^{2 k} A_{i, i+\underline{H}}>0,
$$

and

$$
\sum_{i=2 k+1}^{n-2-\bar{H}} A_{i, i+\bar{H}}^{\prime}=\sum_{i=2 k+1}^{n-1-\bar{H}} A_{i, i+\bar{H}}+1>0+1>0 .
$$

If West has the highest remaining card, then we need only look at the case that $\underline{H}=0$. Then we have

$$
\begin{aligned}
A_{2,2}^{\prime}+\cdots+A_{n-2, n-2}^{\prime}=A_{2,2} & +\cdots+A_{n-2, n-2} \\
& =T_{0}-A_{1,1}-A_{n-1, n-1}-A_{n, n} \geq 1-1-1+1 \geq 0 .
\end{aligned}
$$

We now consider the case that $\bar{H}=0$. Since $n \equiv \bar{H}(\bmod 2), n$ is even. Let $k$ be maximal with

$$
\sum_{i=2}^{2 k} A_{i, i}>0
$$

Then

$$
\sum_{i=2}^{2 k} A_{i, i-1}>0
$$

We have

$$
T_{-1}^{\prime}=\sum_{i=2}^{2 k} A_{i, i-1}+\sum_{i=2 k+1}^{n-1} A_{i, i-1} \geq \sum_{i=2}^{2 k} A_{i, i-1}+\sum_{i=2 k+1}^{n-1} A_{i, i} \geq 1+1>0 .
$$


Hence $\underline{H}^{\prime} \geq-1$ and $\bar{H}^{\prime} \geq 0$.

Suppose that East has the highest remaining card. Then $A_{n-1, n-1}^{\prime}=A_{n-1, n-1}=-1$. We have

$$
\begin{aligned}
A_{2,2}^{\prime}+\cdots+A_{n-2, n-2}^{\prime}=A_{2,2}+\cdots & +A_{n-2, n-2} \\
& =A_{2,2}+\cdots+A_{n-1, n-1}-A_{n-1, n-1} \geq 0+1 \geq 0 .
\end{aligned}
$$

Similarly,

$$
\sum_{i=2}^{2 k} A_{i, i-1}^{\prime}=\sum_{i=2}^{2 k} A_{i, i-1}>0
$$

and

$$
\sum_{i=2 k+1}^{n-2} A_{i, i}^{\prime}=\sum_{i=2 k+1}^{n-2} A_{i, i}=\sum_{i=2 k+1}^{n-1} A_{i, i}^{\prime}-A_{n-1, n-1} \geq 1+1>0 .
$$

Finally suppose that West has the highest remaining card. Then

$$
A_{n-1, n-1}=1 .
$$

By Lemma 9.2, we can assume that $k=n / 2-1$. We have

$$
\sum_{i=2}^{n-2} A_{i, i-1}>0
$$

Suppose that there is an $m$ such that

$$
\sum_{i=2}^{2 m} A_{i, i}^{\prime}<0
$$

and

$$
\sum_{i=2 m+1}^{n-2} A_{i+1, i}^{\prime}<0
$$

Then

$$
\begin{aligned}
& A_{2,2}+\cdots+A_{n-1, n-1}=A_{2,2}^{\prime}+\cdots+A_{n-1, n-1}^{\prime} \\
& =\sum_{i=2}^{2 m} A_{i, i}^{\prime}+\sum_{i=2 m+1}^{n-2} A_{i, i}^{\prime}+A_{n-1, n-1} \\
& \quad \leq \sum_{i=2}^{2 m} A_{i, i}^{\prime}+\sum_{i=2 m+1}^{n-2} A_{i+1, i}^{\prime}+1 \leq-1-1+1<0,
\end{aligned}
$$

contrary to assumption. Hence there is no such $m$, which means that $H^{\prime} \geq 0$. This completes the analysis of Case IV, and thereby the proof of Theorem 9.1. 


\subsection{An optimal strategy}

In the proof of Theorem 9.1, we obtained an optimal strategy for Whist. This strategy is described from West's perspective for each of the eight main cases of the proof. In the remaining cases, where $H=\underline{H}$, the normal strategy is optimal. We do not restate this strategy here, but we make some remarks on when to hold up. It was shown in Lemma 9.3 that there is no point in holding up if the opponent plays his smallest card. The proof of Theorem 9.1 shows something much stronger:

Theorem 9.4. There is no point in holding up if one can take the trick with a card smaller than the ace.

Theorem 9.1 already shows that the ace has a special status. Theorem 9.4 shows that there is no reason to hold up, other than to keep the possession of the ace. However, as the example (14) in Section 7 shows, it is not always correct to keep the ace as long as possible.

As can be seen from the analysis of cases $\mathrm{I}(\mathrm{E})-\mathrm{IV}(\mathrm{E})$, one optimal choice of strategy for playing second when we have the ace and cannot win the trick with a smaller card, is as follows: Win the trick with the ace in Case III if $\underline{H}=0$, in Case IV, and in the cases where $H=\underline{H}$ (which do not belong to any of the cases I-IV). Otherwise hold up.

\section{Computational complexity}

From the point of view of computational complexity, one can say that a game such as whist is solved when there is a polynomial time algorithm that computes the game theoretical value of any given deal. It is clear that the conditions occurring in Theorem 9.1 can all be verified in polynomial time, and that this theorem thereby gives a solution to the game. In this section, we point out that the game theoretical value can be computed more efficiently than by directly applying Theorem 9.1 .

We assume that the deal $D$, which is to be regarded as the input to an algorithm computing the game theoretical value, is represented as a vector $C_{1}, C_{2}, \ldots, C_{2 n}$, where

$$
C_{i}=\left\{\begin{array}{l}
1, \text { if East holds the card } i \\
-1, \text { if West holds card } i
\end{array}\right.
$$

We consider the problem of computing $H(D)$ from the vector $C_{1}, \ldots, C_{2 n}$. We show that this can be done in almost linear time.

Theorem 10.1. There is an algorithm that computes $H(D)$ using a linear number of additions, subtractions, assignments and comparisons of numbers of size $O(n)$. Hence assuming that each operation takes time $O(\log n)$, this algorithm will run in time $O(n \log n)$.

For $i=0, \ldots, 2 n$, let

$$
S(i)=C_{1}+\cdots+C_{i}
$$


We can compute and store $S(1), \ldots, S(2 n)$ using the recursion

$$
S(i+1)=S(i)+C_{i+1}
$$

We now compute and store $T_{k}$, for $-n<k<n$. The following procedure will do this in a linear number of operations. It uses an array of numbers $T[-n+1], T[-n+$ $2], \ldots, T[n-1]$. We will show that the final value of $T[k]$ is equal to $T_{k}$.

1. Initialize. Put

$$
T[-n+1]=1, \quad T[-n+2]=2, \quad \ldots, \quad T[-1]=n-1,
$$

and

$$
T[0]=-n, \quad T[1]=-n+1, \quad \ldots, \quad T[n-1]=-1 .
$$

2. Loop. For $i=1, \ldots, 2 n$ :

if $C_{i}=1$ then

$$
T[S(i-1)]:=T[S(i-1)]-(i-1)
$$

else

$$
T[S(i)]:=T[S(i)]+i .
$$

We now analyze the procedure for computing $T_{k}$. Suppose that in a deal $D_{1}, i-1$ of the smallest $i+j-2$ cards belong to West, and the remaining $j-1$ of these cards belong to East. Then $S(i+j-2)=j-i$. Suppose further that the next card belongs to West, and the following to East, that is, $C_{i+j-1}=-1$ and $C_{i+j}=1$. Then in round $i+j-1$ of the loop, the value of $T[S(i+j-1)]$ will be increased by $i+j-1$, and in round $i+j$, it will be decreased by the same amount. Hence whenever a negative entry is followed by a positive one in the array $C_{1}, \ldots, C_{2 n}$, none of the numbers $T[k]$ will change over the corresponding two rounds of the loop.

Suppose now that the deal $D_{2}$ differs from $D_{1}$ only in that

$$
C_{i+j-1}\left(D_{2}\right)=1
$$

and

$$
C_{i+j}\left(D_{2}\right)=-1
$$

that is, the two cards $i+j-1$ and $i+j$ have been interchanged. If the algorithm above is run with $D_{2}$ as input instead of $D_{1}$, then in round $i+j-1$ of the loop, $T[S(i+j-2)]=T[j-i]$ will be decreased by $i+j-2$, and in the following round, it will be increased by $i+j$, giving a total increase by 2 . Except for these two rounds, the execution of the algorithm will be identical to the one which occurs with $D_{1}$ as input.

Hence with $D_{2}$ as input, the final value of $T[j-i]$ will be greater by 2 than the final value of $T[j-i]$ with $D_{1}$ as input. Since the matrices $A\left(D_{1}\right)$ and $A\left(D_{2}\right)$ differ only in 
position $(i, j)$, where $A_{i, j}\left(D_{1}\right)=-1$ and $A_{i . j}\left(D_{2}\right)=1$, we have $T_{j-i}\left(D_{2}\right)=T_{j-i}\left(D_{1}\right)+2$, and $T_{k}\left(D_{1}\right)=T_{k}\left(D_{2}\right)$ whenever $k \neq j-i$. Hence if the procedure above computes the values of $T_{k}$ for $-n<k<n$ correctly for one of the two deals $D_{1}$ and $D_{2}$, then it also computes these values correctly for the other. Since every deal can be obtained from every other deal by a sequence of swaps of adjacent cards, it now suffices to show that the procedure computes the values of $T_{k}$ correctly for one deal. It was noted above that whenever two adjacent elements of the array $C_{1}, \ldots, C_{2 n}$ are -1 and +1 respectively, no value $T[k]$ will change over the corresponding two rounds of the loop. Therefore, with the deal given by $C_{1}, \ldots, C_{2 n}=-1,1,-1,1, \ldots,-1,1$, that is, the deal for which

$$
A_{i, j}=\left\{\begin{array}{l}
-1, \quad \text { if } i \geq j \\
1, \quad \text { if } i<j
\end{array}\right.
$$

the final values of $T[k]$ for $-n<k<n$ will be the same as those assigned in the initialization. For this deal, these are the correct values of $T_{-n+1}, \ldots, T_{n-1}$.

Having computed $T_{k}$ for $-n<k<n$, we can of course find $\underline{H}$ and $\bar{H}$ in another linear number of operations. Since we have stored all the partial sums of $C_{i}$, we can use the fact that

$$
A_{i, j}=\left\{\begin{array}{l}
1, \text { if } S(i+j-1)>j-i, \\
-1, \text { if } S(i+j-1)<j-i .
\end{array}\right.
$$

to compute and store all the matrix elements $A_{i, j}$ for which $j-i=\underline{H}$ or $j-i=\bar{H}$. We can then recursively compute the sums

$$
\sum_{i=2}^{2 k} A_{i, i+\underline{H}}
$$

and

$$
\sum_{i=2 k+1}^{n-\bar{H}-1} A_{i, i+\bar{H}}
$$

for all values of $k$ for which both sums are nonempty. It is now clear that all the conditions involved in Theorem 9.1 can be checked in $O(n)$ operations. This proves Theorem 10.1.

Acknowledgment. The author thanks Klas Mårtensson for providing the reference [8], and Charles Magri for some valuable comments on an earlier draft of the paper.

\section{References}

[1] E. Berlekamp, J. H. Conway, and R. Guy, "Winning ways for your mathematical plays" I-II, Academic Press, New York 1982.

[2] J. Kahn, J. C. Lagarias, and H. S. Witsenhausen, Single-suit two-person card play, Internat. J. Game Theory 16 (1987), 291-320. 
[3] J. Kahn, J. C. Lagarias, and H. S. Witsenhausen, Single-suit two-person card play II: Domination, Order 5 (1988), 45-60.

[4] J. Kahn, J. C. Lagarias, and H. S. Witsenhausen, Single-suit two-person card play III: The misère game, SIAM J. Disc. Math. Vol. 2 No. 3 (1989) 329-343.

[5] E. Lasker, "Das verständige Kartenspiel", Berlin 1929.

[6] E. Lasker, "Encyclopedia of Games", Vol. I, Card Strategy, E. P. Dutton \& Co., New York 1929.

[7] G. Ottlik and H. Kelsey, "Adventures in card play", Victor Gollancz Ltd in association with Peter Crawley, London 1979.

[8] D. Parlett, "A History of Card Games", Oxford University Press, 1991.

[9] J. Wästlund, Two-person symmetric whist, The Electronic Journal of Combinatorics 12 (2005), \#R44. 